\title{
The additional value of lower respiratory tract sampling in the diagnosis of COVID-19. A single centre real life observational study.
}

\section{Luca Morandi ( $\nabla$ mmlcu2@unife.it )}

Respiratory Medicine, Emergency Department, Azienda Ospedaliero Universitaria di Ferrara

Francesca Torsani

Respiratory Medicine, Emergency Department, Azienda Ospedaliero Universitaria di Ferrara

Giacomo Forini

Respiratory Medicine, Emergency Department, Azienda Ospedaliero Universitaria di Ferrara

\section{Marianna Padovani}

Respiratory Medicine, Department of Translational Medicine, University of Ferrara,

\section{Aldo Carnevale}

Radiology Department, Azienda Ospedaliero Universitaria di Ferrara

\section{Anna Pecorelli}

Radiology Department, Azienda Ospedaliero Universitaria di Ferrara

\section{Marco Piattella}

Respiratory Medicine, Emergency Department, Azienda Ospedaliero Universitaria di Ferrara Ippolito Guzzinati

Respiratory Medicine, Emergency Department, Azienda Ospedaliero Universitaria di Ferrara

\section{Alberto Papi}

Respiratory Medicine, Department of Translational Medicine, University of Ferrara,

Marco Contoli

Respiratory Medicine, Department of Translational Medicine, University of Ferrara,

\section{Research Article}

Keywords: SARS-CoV-2, diagnosis, bronchoscopy, blind nasotracheal aspiration, Chest CT

Posted Date: February 26th, 2021

DOI: https://doi.org/10.21203/rs.3.rs-212656/v1

License: (c) (1) This work is licensed under a Creative Commons Attribution 4.0 International License. Read Full License 


\section{Abstract}

Background: Since December 2019 SARS-CoV-2 has been identified as the causal agent for severe cases of pneumonia in China and it spread all over the world, putting great pressure on Health Systems. Despite regarded as the standard procedure, sensitivity of nasopharyngeal swab (NPS) is suboptimal. In clinical/radiological suspicion of SARS-CoV-2 infection despite negative NPS test, other tests may be required to rule out the infection.

Objectives: To evaluate correlations between lower respiratory tract (LRT) testing for SARS-CoV-2 in suspect patients with 2 negative NPSs and clinical symptoms, laboratory values and the CT scan review score by the Radiological Society of North America.

Method: Retrospective analysis of radiological, clinical and laboratory data from patients who underwent LRT sampling (blind nasotracheal aspiration or bronchial washing) for suspected COVID-19 after 2 negative NPS. Chest CTs were reviewed by two blinded radiologist using the Radiological Society of North America score for analysis purpose.

Results: SARS-CoV-2 was detected in 7 out of 59 patients (11.9\%). No clinical or laboratory value were found to correlate with positive test. CT scan showed good sensitivity $(71.4 \%)$ and accuracy $(81.3 \%)$, with a very high NPV (95.7\%). Features scored as 'typical' showed good correlation with SARS-CoV2 detection on LRT. No complications nor staff's infections were reported.

Conclusions: In suspect cases with at least 2 negative swabs CT scan revision can be helpful in ruling out SARS-CoV-2 infection. In selected cases lower respiratory tract sampling can help in confirming COVID-19 in cases with consistent CT features.

\section{Introduction}

In late December 2019 a cluster of pneumonia of unknown etiology was detected in the area of Wuhan, China [1]. The responsible agent was identified as a coronavirus first named nCoV19, subsequently changed in SARS-CoV-2 in accordance to genetic similarity with the coronavirus known as etiologic agent of the Severe Acute Respiratory Syndrome in 2003 (SARS-CoV-1) [2]. Since then, the epidemic spread through all the continents, until the pandemic was declared by the WHO on March 11th, 2020 [2,3]. SARSCoV-2 infection (COVID-19) has been shown to cause a wide spectrum of syndromes, varying from unapparent, asymptomatic course and pauci-symptomatic infection mainly involving the upper respiratory tract and/or the GI system, to pneumonia and severe adult respiratory distress syndrome requiring intensive care support, possibly leading to multi-organ failure and even death $[2,4]$. The diagnosis is based on molecular techniques for the identification of $\mathrm{N}$ and $\mathrm{E}$ genes of SARS-CoV-2 genome by RT-PCR reaction [5]. The nasopharyngeal swab (NPS) is the recommended sample for the assay [5]. However, in clinical practice the performance of NPS RT-PCR is suboptimal, with a reported sensitivity of about $66 \%$ [6]. Several laboratory biomarkers, including lymphocyte count, D-dimer assay, and troponin I levels have been correlated to disease prognosis [4] but none of them provided sufficient 
diagnostic specificity $[2,4,7,8]$. High resolution computed tomography (CT) scan of the chest can reach very high sensitivity, for the diagnosis of COVID19 [9]. Some specific radiological pneumonia findings (graded as typical vs indeterminate vs atypical CT imaging features) have been identified to support the diagnostic process [10]. It is not that uncommon to experience cases of patients presenting with suggestive clinical symptoms (mainly consisting of fever, cough and dyspnea) and suggestive but not conclusive radiological findings, and negative NPS RT-PCR test. Repetition of the test is highly recommended in these patients, given the epidemiological implications of allocating a SARS-CoV-2 infected case to an inappropriate setting. Still, even double negative result might not be sufficiently specific for ruling out COVID-19. In such cases, respiratory physicians may therefore be asked to help in the diagnostic process by sampling the lower airways through bronchoscopy. Indeed, lower respiratory tract (LRT) sampling through bronchial endoscopy with either bronchoalveolar lavage (BAL) or bronchial washing (BW) has been shown to be useful in etiologic definition of pneumonias, even in case of viral pneumonia $[11,12]$. For SARS-CoV-2 infection, PCR assay performed on BAL has shown very high sensitivity [6]. However, concerns have been raised on the procedure related biological risk for the HCP involved . In fact, the American Association of Bronchology and Interventional Pulmonology (AABIP) discourages routine use of bronchoscopy in suspect cases [13]. Other associations released similar position papers/recommendations during the early phase of the epidemic [14]. To explore the potential value of LRT sampling on top of the clinical, laboratory and CT imaging information in subjects with a suspect of SARS-CoV-2 infection and at least 2 negative NPS, we performed a retrospective analysis of the diagnostic yield of lower respiratory tract (LRT) sampling in patients with suspected COVID-19 because of the clinical presentation and/or radiological findings but negative NPS for SARS-CoV-2 molecular detection. Moreover we aimed to assess the diagnostic values of respiratory symptoms, blood biomarkers and HRCT findings in these patients/setting.

\section{Materials And Methods}

\section{Study design and population}

This is an observational, retrospective, single center study. The analysis included all admitted patients between March and September 2020 with the following:

- clinically suspected SARS-CoV-2 infection as per WHO recommendations (defined as respiratory symptoms of acute onset or contact with a confirmed case)

- confirmed chest radiological abnormalities suspicious (but not conclusive) for interstitial pneumonia

- at least 2 negative NPSs

- LRT sampling for SARS-CoV-2 RT-PCR test

Patients with only 1 negative NPS and patients with no CT scan available were excluded. The LRT samplings were performed by blind nasotracheal aspiration (BNTA) with a 14 Ch diameter suction 
catheter, or with bronchial endoscopy through bronchial washing (BW). Bronchoscopy was performed either with Pentax FB18V/FB15V fibreoptic bronchoscope (Pentax ${ }^{\circledR}$, Japan) or a single use aScope ${ }^{\mathrm{TM}} 4$ Broncho flexible bronchoscope (Ambu ${ }^{\circledR}$, Denmark). The decision on which test to perform (BNTA vs BW) was taken by the endoscopists in multidisciplinary discussion with the referring clinician, based on clinical history review, clinical conditions/performance status, and extension of the alterations on chest CT. The samplings were performed in protected environment by expert personnel according to international recommendations $[15,16]$. The study was approved by the Local Ethic Committee and informed consent was collected. The study was conducted ethically in accordance with the World Medical Association Declaration of Helsinki.

\section{Aims}

To retrospectively evaluate the diagnostic yield of LRT sampling in patients with repeated negative NPS for SARS-COV2 infections but clinical and/or radiological suspicion for COVID-19. Secondary aims included correlations between laboratory biomarkers, symptoms, radiological findings at HRCT scan and SARS-CoV-2 infection detected on LRT sampling.

\section{Analysis and procedures}

SARS-CoV-2 detection on LRT samplings was retrospectively reviewed. The threshold for positive result was set at 40 or less RT-PCR cycles.

We also analyzed clinical records to evaluate whether reported symptoms, laboratory biomarkers and CT scan findings can be useful in the diagnostic process. For this purpose, we set a three dimensional score to grade clinical, laboratory and radiological data.

The clinical score considered the 3 most relevant symptoms (cough, dyspnea and fever). Given the epidemic phase of the disease at the time of study conduction, close contact with a confirmed case was not considered necessary to suspect SARS-COV-2 infection in presence of suggestive symptoms. We also included in the clinical score respiratory failure which is often present in COVID-19 patients at hospital admission. Respiratory failure was defined as $\mathrm{PaO} 2<60 \mathrm{mmHg}$ in ambient air on blood gas analysis, or SpO2 $<94 \%$ in ambient air. We assigned score 1 to presence and 0 to absence of each of the 4 items.

Given the retrospective nature of the study, we included in the lab score variables that were considered clinically relevant from a literature review and were available for most of the patients included in the study: lymphocyte count, D-dimer assay, PCR and LDH. We arbitrarily set a 4 degree level score for lymphocyte count ( $0=$ normal: $>1500$ cells $\mu \mathrm{L}, 1=$ mild reduction: between 1000 and $1500 / \mu \mathrm{L}, 2=$ moderate reduction: between 500 and $1000 / \mu \mathrm{L}, 3=$ severe reduction: $<500 / \mu \mathrm{L}$ ), and a 2 level score for the remaining variables ( $0=$ normal, $1=$ increased: $>U L N$ for $L D H,>500 \mathrm{ng} / \mathrm{mL}$ for $\mathrm{D}$-dimer and $>0.5 \mathrm{mg} / \mathrm{dL}$ for $P C R)$.

For the purpose of our study we needed to objectively identify suspect imaging features. Therefore, we had the high resolution chest CT scans blindly reviewed by two radiologist, and agreed to score imaging features according to the Radiology Association of North America score [10]: $0=$ no pneumonia, $1=$ 
atypical features for COVID-19, 2= indeterminate features for COVID-19, 3= Typical features for COVID-19 pneumonia. Cases in which there was a disagreement between the two radiologists, were reviewed and solved by consensus. To evaluate the diagnostic performance, we considered as positive only CTs with a score 3. CTs with score 0,1 and 2 were considered as negative. Diagnostic accuracy was defined as the ratio between all cases in which CT score correctly assigned patients to either positive or negative for SARS-CoV-2 infection and the total number of patients. The complete scoring system adopted is resumed in Figure 1.

We looked for correlations between clinical, laboratory, radiology and combined score and LRT test result, which represented our diagnostic gold standard.

\section{Statistics}

All parametric and non parametric variables are presented as mean and range. Statistical significance was set at $95 \% \mathrm{Cl}$ with $\mathrm{p}$ value $<0.05$. Student's t-test and Mann-Whitney $\mathrm{U}$ test were used to compare characteristics between LRT positive and negative patients, as appropriate. Multivariate analysis to explore correlations between clinical, laboratory, CT and combined score was also performed. Data analysis was performed through Graph Pad Prism ( San Diego, CA (US), version 7, and through IBM $®$

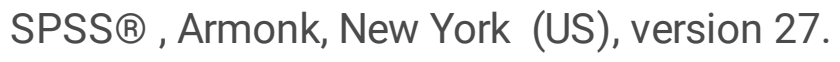

\section{Results}

\section{Study Population}

Between March $26^{\text {th }}$ and September $14^{\text {th }}, 67$ patients underwent LRT sampling either through bronchoscopy or BNTA after 2 negative NPSs for suspect SARS-CoV-2 infection. Eight patients were excluded from the analysis because of the following reasons: 7 patients underwent only 1 NPS before the bronchoscopy; 1 patients did not undergo CT scan before the bronchoscopy.

The characteristics of the study population $(n=59)$ are reported in Table 1. Mean age resulted $70.6 \mathrm{y}$, most of the patients were male $(72.9 \%)$. Thirty-four patients $(57.6 \%)$ had respiratory failure at presentation. All the patients had lung abnormalities in CT scans. Data were missing for 2 patients for lymphocyte count, 20 patients for D-dimer, 9 patients for LDH and 5 patient for PCR. After revision, presence of CT scan alterations consistent with lung infiltrates were confirmed in 56/59 cases (94.9\%).

LRT sampling was performed with BNTA in 15 patients (28\%), while endoscopy was performed in 44 (72\%). SARS-CoV-2 was identified in LRT samples from 7 cases ( $11.9 \%$ of the included population). These included 5 bronchial washings and 2 BNTAs. Negative test was obtained in the remainder 52 patients.

\section{Positive vs negative patients}


Table 2 shows comparison of characteristics and scores between positive and negative patients. Positive cases were significantly younger, with no significant difference in gender distribution as compared to negative patients. Among positive patients, a higher percentage presented with cough. However, no statistically significant differences in symptoms prevalence was detected. No statistically significant difference was found in the laboratory values between SARS-CoV-2 positive and negative patients, with a trend for decreased blood lymphocytes count in COVID-19 subjects.

\section{Score Results}

Table 3 summarizes the score distribution in the whole population analyzed and the comparison between positive and negative patients.

Only 6 patients were negative at the clinical evaluation (10.2\%), with 2 patients showing a clinical score of 4 (3.4\%). The mean clinical score was 1.89 (0-3) in SARS-CoV-2 negative patients and $2.14(0-4)$ in SARS-CoV-2 positive patients, with no statistically significant difference.

All patients presented with at least one laboratory abnormality, the most common being increased PCR, with mean scores of 3.15 and 3.17 in the SARS-CoV-2 negative and positive group, respectively, with no statistically significant difference.

After revision by two blinded radiologists, 13 chest CTs (22.0\%) received a score of 3 (meaning consistent with COVID-19), 26 (44.1\%) were scored 2 (indeterminate for COVID-19), 17 (28.8\%) were scored 1 (not typical for COVID-19) and the remaining 3 (5.1\%) were scored 0 (no pneumonia). Five out of the 13 typical CT cases proved positive at LRT testing. The remaining 2 patients with positive LRT test were given a CT score of 2 and 1, respectively. The consequent diagnostic performance of the CT scan, as shown in Table 4, resulted in a sensitivity of $71.4 \%$, a specificity of $84.6 \%$ and a positive predictive value and negative predictive value of $38.5 \%$ and $95.7 \%$, respectively. Diagnostic accuracy resulted $83.1 \%$. Revision of the clinical data revealed that the 2 patients with false negative CT score were re-admitted after previous hospitalization for COVID-19 and had been discharged after clinical resolution and two consecutive negative NPSs as per local protocol. Reason for re-admission in both cases was sudden appearance of respiratory symptoms and fever. A statistically significant difference was detected in the CT revision score, showing higher values in positive cases (mean 2.57, range 1-3) as compared to the negative ones (mean 1.73, range $0-3, p 0.01$ ). No statistically significant correlations were detected between the total score (sum of clinical, laboratory and radiological score) and positivity for SARS-CoV-2 (mean score 6.82 vs 7.62 for negative and positive patients, respectively, p 0.34).

Multivariate analysis (as shown in Table 5) confirmed a positive correlation between CT score and SARSCoV-2 infection, while an inverse correlation was found between age and likelihood of SARS-CoV-2 infection. No correlations were detected with clinical and laboratory variables.

\section{Microbiological analysis on LRT sampling}


LRT sampling resulted in isolation of pathogens other than SARS-COV-2 in 25 out of 59 cases (42.4\%). Microbiological analysis was not performed on 12 cases, all consisting of BNTA, due to less material collected. Details on isolated pathogens are provided in Table 6. To be noted, in 17 (28.8\%) cases a mycotic infection was detected, with Candida Spp. as the most common agent. Among bacteria, Gram negative Spp were most frequently isolated, P. Aeruginosa being the most consistently isolated.

\section{Complications}

Five complications (8.5\%) were reported, mainly consisting in drop in peripheral blood oxygen saturation during the procedure. In most cases, no specific intervention was required, with spontaneous resolution after completion of the exam. In two cases the drop in oxygen saturation prompted early termination of bronchoscopy, and in one out of two it required administration of high flow oxygen and i.v. steroids to resolve. All patients with reported complications were already in oxygen supply at the beginning of the procedure, and in the most severe case pulmonary embolism was following detected at a contrast enhanced CT scan, accounting for higher procedure risks. No complications were reported with BNTA procedures. No site personnel was infected by SARS-CoV-2 as a consequence of the procedures.

\section{Discussion}

This study aimed to explore the relationship between clinical, laboratory and radiological characteristics of a cohort of patients undergoing LRT sampling due to suspect SARS-CoV-2 infection, after at least two negative NPSs.

We found 7 positive cases out of $59,11.9 \%$ of the analyzed population. We did not find any relevant relationship between clinical symptoms at admission and positivity of LRT samples, nor between any of the laboratory values considered (lymphocyte count, D-dimer assay, LDH and PCR) and SARS-CoV-2 diagnosis in LRT samples.

Revision of the CT scans by two blinded radiologists showed a sensitivity as high as $71.4 \%$ with a diagnostic accuracy of $81.3 \%$ using the Radiological Society of North America score. A very high negative predictive value was also detected (95.7\%).

Other studies explored the role of bronchoscopy in COVID-19, with heterogeneous populations, patients selection criteria and conclusions. Ora et al studied BAL performance in SARS-CoV-2 search in patients with suspect COVID-19 based on symptoms and CT scan, at least two negative swabs and negative serologic test for SARS-CoV-2. They report no detection of SARS-CoV-2, and conclude that in such cases BAL should not be considered for testing [17]. Geri et al also used BAL to search for SARS-CoV-2 on 79 consecutive patients admitted to respiratory units of three Italian hub hospitals, finding high concordance for negative result (97.5\%) between NPS and BAL. The Authors suggest that BAL has a limited role in the diagnosis of COVID19 when both thoracic imaging and upper respiratory swabs are negative [18]. However, only 50 out of 79 patients had at least two negative swabs, which would seem more prudent to spare health workers from exposure in unnecessary aerosol generating procedures, as per international 
recommendations. Furthermore, only 63 patients had a CT scan, and only 38 of them showed signs compatible with viral infection. Most importantly, the 2 patients with positive BAL had highly compatible CT scan for COVID19. Mondoni et al assessed the yield of both BAL and BW in 78 patients from six different Italian Hospitals, who underwent bronchoscopy for SARS-CoV-2 search for suspicion of COVID19 despite two negative NPSs. The Authors report a very high prevalence of COVID-19 (55.1\%) and report no significant differences between BAL and BW [19]. More recently, another Italian multicenter study by Patrucco et al isolated SARS-CoV-2 on BAL in 43/131 (32.8\%) patients [20]. Included patients differed in key characteristics, such as number of pre-test negative NPSs, suspicion of infection and indications to perform a bronchoscopy. High agreement is reported in this work between NPSs and BAL especially in negative samples, but on the other hand only 10 out of 43 positive patients had a positive NPS. Most of the included patients presented chest CT alterations. A subsequent review of the CT scan in 46 patients from this cohort using international societies recommended scores showed good correlation between suggestive features and BAL positivity for SARS-CoV-2 for both the CO-RADS and the Radiological Society of North America score [21].

We included in our analysis suspected patients with at least 2 consecutive negative NPSs. We included BNTA as a sampling technique for its less invasive nature and ease of performance even for less trained personnel. At the time of analysis, serology for SARS-CoV-2 was not available in our hospital setting to rule out infection with SARS CoV2. Hence, the conclusion of the work from Ora et al do not apply to our cohort. In fact, not performing LRT sample analysis would have led to 7 missed cases, with possible great risk of in-Hospital spreading of the infection.

The potential of the CT of the thorax in the diagnosis of COVID-19 has been first suggested by Ai et al who, after reviewing more than 1000 CT scans from Chinese patients, calculated a sensitivity of $97 \%$ for SARS-CoV-2 associated pneumonia. Since then, knowledge on radiological appearance of SARS-CoV-2 related lung alterations has increased, leading to the development of suggested standardized report systems $[10,22]$.

However, the diagnostic performance of CT scan not merely depends on the prevalence of the disease in the studied population, but is also affected by non-specific findings of COVID-19 that overlap with those of other conditions, resulting in a substantial rate of false-positive examinations [23]. A Cochrane metaanalysis by Salameh et al including 78 studies evaluating CT scan on 8105 participants found a $93.1 \%$ sensitivity for studies including confirmed cases and a $86.2 \%$ sensitivity in studies including suspected cases. However, specificity resulted much lower (18.1\%) [24]. Another meta-analysis by Kovacs et al reported sensitivity between 67 and 100\% for chest CT. Interestingly, the Authors notice that the sensitivity of NPS test clearly influences the calculated CT scan specificity, which may therefore be underestimated. Korevaar et al retrospectively evaluated the added value of chest CT scan analyzed with the CO-RADS score to RT-PCR testing [22]. The Authors found a CT score consistent with COVID19 in $92.9 \%$ of the patients with initial positive RT-PCR test. They also reported that 13 cases out of 38 with positive CT but negative NPS test ultimately had confirmed SARS-CoV-2 infection after repetition of RT-PCR testing. As in previous reports, specificity was lower, being only $25 \%$ [25]. 
Our data further support the importance of chest CT in COVID19 diagnosis, especially when a standardized scoring system is applied. We had our CT scans reviewed by two radiologists, finding good sensitivity, specificity and NPV resulting in promising diagnostic accuracy. Not to overestimate CT performance, we included in the reported calculation 2 patients who already had been admitted and treated with a COVID-19 diagnosis, and had been discharged from hospital with negative RT-PCR on NPS. It has already been described that BAL retains RT-PCR positivity for longer than NPS [26], though it is not clear whether this positivity really reflects infectivity. The CT features for these 2 cases may not represent the acute phase of the disease, therefore misleading the reading radiologist [27]. By excluding these two patients from the analysis, CT sensitivity would have resulted $100 \%$, and diagnostic accuracy would have been $86 \%$. Certainly, the low incidence of infection in our population, also as a consequence of considering only patients with 2 negative NPSs, lead to a higher calculated specificity and NPV, which may not be applicable to other scenarios.

Our results are consistent with published works on the topic, confirming the overall good concordance between negative CT findings, negative NPSs and negative LRT RT-PCR testing. An important finding of this study is that even 2 negative NPSs cannot safely rule out SARS-CoV-2 infection, especially when typical features are detected on CT scan, as reported by other groups $[18,19,21]$. Data also seem to suggest that since most symptoms are overlapping in different diseases, they should not be considered disease specific per se, and confirm that probably most of the interest for lab values stands in their predictive value for clinical outcomes $[2,4,8]$.

In this scenario, having the CT scan revised and scored according to an objective and repeatable score is key in interpreting imaging features, as imaging revision by respiratory clinician would have led to a greater number of false positives, given the presence of ground glass opacities in most of the cases included.

Our work suggests bronchial washing to be as effective as BAL. In selected cases, even BNTA can be a valuable choice, with lower risks for complications, considering the high prevalence of pre-examination respiratory failure in this population.

In fact we only report five complications during BW sampling, none severe and all consisting in drop in oxygen saturation during sample collection.

Ora et al and Patrucco et al found alternative specific infective agents in a significant proportion of their patients $[17,20]$. In our cohort BW identified respiratory infection by either bacteria or fungi in more than $40 \%$ of the included population, with overlapping bacterial and fungal infection in $13.6 \%$. This confirms the high value of LRT sampling in managing lung infections, potentially leading to clinical changes in terms of antibiotic choice.

Our study certainly has all the limitations connected with its retrospective design. Other limitations include its mono centric nature and the relatively small number of cases analyzed. Data on specificity and NPV should be weighted with regards to the low prevalence of infection we found in this population. 
However, our study clearly reflects real life clinical scenario in a hub University Center during the pandemic phase of the infection, where promptly confirming SARS-CoV-2 infection is mandatory to avoid in-hospital spreading of the disease. The lower incidence of SARS-CoV-2 detection in the lower respiratory tract as compared to that of Mondoni et al and Patrucco et al, probably reflects differences in epidemiologic prevalence of the infection between different areas. To the best of our knowledge, we are the first to assess a repeatable combined clinical, laboratory and radiological score in the diagnostic workup of COVID-19, despite only showing good correlations with CT scan.

Our data suggest that COVID-19 is a multi-modal diagnosis which should not rely on only one test, but rather on a panel of tests, which to the best of our knowledge should include CT scan and LRT samples, when CT scan is consistent and less invasive tests cannot be conclusive. A multi-disciplinary discussion should be advised in more complex cases, including at least radiologists and pulmonologists. Our data also show that bronchoscopy, when protective equipment is used as indicated in several reports, can be performed preserving health workers' safety. It must be noticed that SARS-CoV-2 diagnosis is not only necessary for preventing spreading of the infection, but also for treatment access (e.g. participation in clinical trials), and for safety reasons before undertaking immunosuppressive or chemotherapy treatment. Another application of bronchoscopy in suspected cases can include differential diagnosis with other infectious diseases.

\section{Conclusion}

In the most challenging scenarios, i.e. when clinical data, laboratory analysis and RT-PCR test on NPS are unable to lead to a definite confirmation or exclusion of SARS-CoV-2 diagnosis, our data suggest that CT scan revision and lower respiratory tract sampling represent useful tools. We suggest multi disciplinary discussion involving at least a radiologist and a respiratory physician in the evaluation of LRT sampling opportunity, considering the potential risks for health care workers. Ideally, larger multi centric prospective clinical trials with a clear and reproducible definition of positive and negative imaging features will be required to design diagnostic algorithms that maximize the risk/benefit balance for patients and health care workers dealing with COVID-19.

\section{List Of Abbreviations}

SARS-CoV-2 = severe acute respiratory syndrome coronavirus 2

RT-PCR = real time polymerase chain reaction

LRT $=$ lower respiratory tract

$\mathrm{BW}=$ bronchial washing

BNTA = Blind nasotracheal Aspiration 
NPS = nasopharyngeal swab

COVID-19 = Coronavirus Disease 19

$\mathrm{ER}=$ Emergency Room

\section{Declarations}

\section{Ethics approval and consent to participate}

The study was approved by the Local Ethic Committee (Comitato Etico Indipendente di Area Vasta Emilia Centro, CE-AVEC), protocol name BALCOV2, protocol code 825/2020/Oss/AOUFe. The study was conducted ethically in accordance with the World Medical Association Declaration of Helsinki. Informed consent was obtained from all participants, in accordance to the local Ethic Committee.

\section{Consent for publication}

Not Applicable.

\section{Availability of data and materials}

The raw data used/analyzed in the current study are available, upon reasonable request to the corresponding Author and Ethics Committee (CE-AVEC).

\section{Competing Interests}

Dr Morandi L, reports personal fees from Boehringer Ingelheim, outside the submitted work. Dr Contoli reports grants, personal fees and non-financial support from Chiesi, personal fees and non-financial support from AstraZeneca, personal fees and non-financial support from Boehringer Ingelheim, personal fees and non-financial support from Alk-Abello, grants, personal fees and non-financial support from GlaxoSmithKline, personal fees and non-financial support from Novartis, personal fees and non-financial support from Zambon, grants from University of Ferrara - Italy, outside the submitted work;

Dr Papi A reports grants, personal fees, non-financial support from GlaxoSmithKline, AstraZeneca, Boehringer Ingelheim, Chiesi Farmaceutici TEVA and Sanofi/Regeneron; personal fees, non-financial support from Mundipharma, Zambon, Novartis; grants, personal fees and non-financial support from Menarini; personal fees from Roche, grants from Fondazione Maugeri, grants from Fondazione Chiesi, personal fees from Edmondpharma, outside the submitted work.

Drs Torsani F, Forini G, Padovani M, Carnevale A, Pecorelli A, Piattella M, Guzzinati I report no conflict of interest for the present work.

\section{Funding}

The Authors do not report any funding or sponsorship for the present study. 


\section{Authors' Contributions}

Morandi $\mathrm{L}$ and Contoli $\mathrm{M}$ contributed in study design, data analysis and interpretation and manuscript writing, Carnevale A and Pecorelli A reviewed in CT scans review and score. Carnevale A and Papi A also contributed in the review of the manuscript and interpretation of the results. Morandi L, Torsani $F$, Padovani M, Piattella M, Guzzinati I and Forini G contributed in patient enrolling and data collection. All Authors approved final version of the manuscript.

\section{Acknowledgements}

The Authors thank all nursing and sanitary personnel who assisted in the procedures, for their substantial contribution in the realization of the present work.

\section{References}

1. Zhu N, Zhang D, Wang W, Li X, Yang B, Song J, et al. A Novel Coronavirus from Patients with Pneumonia in China, 2019. N Engl J Med. 2020 20;382(8):727-33.

2. Hu B, Guo H, Zhou P, Shi Z-L. Characteristics of SARS-CoV-2 and COVID-19. Nat Rev Microbiol. 2020 Oct 6;1-14.

3. WHO. WHO Timeline - Covid-19 [Internet]. 2020 [cited 2020 Jun 26]. Available from: https://www.who.int/news-room/detail/27-04-2020-who-timeline--covid-19

4. Zhou F, Yu T, Du R, Fan G, Liu Y, Liu Z, et al. Clinical course and risk factors for mortality of adult inpatients with COVID-19 in Wuhan, China: a retrospective cohort study. The Lancet. 2020 Mar 28;395(10229):1054-62.

5. WHO. Laboratory testing for 2019 novel coronavirus (2019-nCoV) in suspected human cases Interim guidance- [Internet]. 2020 [cited 2020 Jun 26]. Available from: https://www.who.int/publications/i/item/10665-331501

6. Wang W, Xu Y, Gao R, Lu R, Han K, Wu G, et al. Detection of SARS-CoV-2 in Different Types of Clinical Specimens. JAMA. 2020 Mar 11;

7. Rello J, Storti E, Belliato M, Serrano R. Clinical phenotypes of SARS-CoV-2: Implications for clinicians and researchers. Eur Respir J [Internet]. 2020 Jan 1 [cited 2020 Nov 12]; Available from: https://erj.ersjournals.com/content/early/2020/04/20/13993003.01028-2020

8. Pourbagheri-Sigaroodi A, Bashash D, Fateh F, Abolghasemi H. Laboratory findings in COVID-19 diagnosis and prognosis. Clin Chim Acta. 2020 Nov 1;510:475-82.

9. Ai T, Yang Z, Hou H, Zhan C, Chen C, Lv W, et al. Correlation of Chest CT and RT-PCR Testing for Coronavirus Disease 2019 (COVID-19) in China: A Report of 1014 Cases. Radiology. 2020;296(2):E32-40.

10. Simpson S, Kay FU, Abbara S, Bhalla S, Chung JH, Chung M, et al. Radiological Society of North America Expert Consensus Statement on Reporting Chest CT Findings Related to COVID-19. 
Endorsed by the Society of Thoracic Radiology, the American College of Radiology, and RSNA Secondary Publication. J Thorac Imaging. 2020 Jul;35(4):219-27.

11. Garbino J, Soccal PM, Aubert J-D, Rochat T, Meylan $P$, Thomas $Y$, et al. Respiratory viruses in bronchoalveolar lavage: a hospital-based cohort study in adults. Thorax. 2009 May;64(5):399-404.

12. Munigala S, Burnham C-AD, Anderson NW, Liang SY, Lawrence SJ, Warren DK. Diagnostic Performance of Multiplex Nucleic Acid Testing of Bronchoalveolar Lavage and Bronchial Wash Specimens for Respiratory Viral Pathogens. J Clin Microbiol [Internet]. 2018 Sep 25 [cited 2020 Nov 23];56(10). Available from: https://www.ncbi.nlm.nih.gov/pmc/articles/PMC6156318/

13. Wahidi MM, Lamb C, Murgu S, Musani A, Shojaee S, Sachdeva A, et al. American Association for Bronchology and Interventional Pulmonology (AABIP) Statement on the Use of Bronchoscopy and Respiratory Specimen Collection in Patients With Suspected or Confirmed COVID-19 Infection. J Bronchol Interv Pulmonol [Internet]. 2020 Mar 18 [cited 2020 Jun 26]; Available from: https://www.ncbi.nlm.nih.gov/pmc/articles/PMC7141581/

14. Lentz RJ, Colt $H$. Summarizing societal guidelines regarding bronchoscopy during the COVID-19 pandemic. Respirol Carlton Vic. 2020;25(6):574-7.

15. Vergnon J-M, Trosini-Desert V, Fournier C, Lachkar S, Dutau H, Guibert N, et al. Bronchoscopy use in the COVID-19 era. Respir Med Res. 2020 Nov;78:100760.

16. Wahidi MM, Shojaee S, Lamb CR, Ost D, Maldonado F, Eapen G, et al. The Use of Bronchoscopy During the Coronavirus Disease 2019 Pandemic. Chest. 2020 Sep;158(3):1268-81.

17. Ora J, Puxeddu E, Cavalli F, Giorgino FM, Girolami A, Chiocchi M, et al. Does bronchoscopy help the diagnosis in Covid-19 infection? Eur Respir J [Internet]. 2020 Jan 1 [cited 2020 Oct 11]; Available from: https://erj.ersjournals.com/content/early/2020/06/08/13993003.01619-2020

18. Geri P, Salton F, Zuccatosta L, Tamburrini M, Biolo M, Busca A, et al. Limited role for bronchoalveolar lavage to exclude Covid-19 after negative upper respiratory tract swabs: a multicenter study. Eur Respir J [Internet]. 2020 Jan 1 [cited 2020 Oct 11]; Available from: https://erj.ersjournals.com/content/early/2020/08/03/13993003.01733-2020

19. Mondoni M, Papa GFS, Rinaldo R, Faverio P, Marruchella A, D'Arcangelo F, et al. Utility and safety of bronchoscopy during SARS-CoV-2 outbreak in Italy: a retrospective, multicenter study. Eur Respir J [Internet]. 2020 Jan 1 [cited 2020 Nov 17]; Available from: https://erj.ersjournals.com/content/early/2020/08/06/13993003.02767-2020

20. Patrucco F, Albera C, Bellocchia M, Foci V, Gavelli F, Castello LM, et al. SARS-CoV-2 Detection on Bronchoalveolar Lavage: An Italian Multicenter experience. Respir Int Rev Thorac Dis. 2020;99(11):970-8.

21. Patrucco F, Carriero A, Falaschi Z, Pasché A, Gavelli F, Airoldi C, et al. COVID-19 Diagnosis in Case of Two Negative Nasopharyngeal Swabs: Association between Chest CT and Bronchoalveolar Lavage Results. Radiology. 2021 Jan 5;203776.

22. Prokop $M$, van Everdingen W, van Rees Vellinga T, Quarles van Ufford $H$, Stöger $L$, Beenen $L$, et al. CORADS: A Categorical CT Assessment Scheme for Patients Suspected of Having COVID-19-Definition 
and Evaluation. Radiology. 2020 Apr 27;296(2):E97-104.

23. Kim H, Hong H, Yoon SH. Diagnostic Performance of CT and Reverse Transcriptase Polymerase Chain Reaction for Coronavirus Disease 2019: A Meta-Analysis. Radiology. 2020 Apr 17;296(3):E145-55.

24. Salameh J-P, Leeflang MM, Hooft L, Islam N, McGrath TA, Pol CB, et al. Thoracic imaging tests for the diagnosis of COVID-19. Cochrane Database Syst Rev [Internet]. 2020 [cited 2020 Nov 17];(9). Available from: https://www.cochranelibrary.com/cdsr/doi/10.1002/14651858.CD013639.pub2/full

25. Korevaar DA, Kootte RS, Smits LP, Aardweg JG van den, Bonta PI, Schinkel J, et al. Added value of chest CT in suspected COVID-19: an analysis of 239 patients. Eur Respir J [Internet]. 2020 Jan 1 [cited 2020 Oct 2]; Available from:

https://erj.ersjournals.com/content/early/2020/06/25/13993003.01377-2020

26. Sethuraman N, Jeremiah SS, Ryo A. Interpreting Diagnostic Tests for SARS-CoV-2. JAMA. 2020 Jun 9;323(22):2249-51.

27. Zhou S, Zhu T, Wang Y, Xia L. Imaging features and evolution on CT in 100 COVID-19 pneumonia patients in Wuhan, China. Eur Radiol. 2020 May 4;1-9.

\section{Tables}

Tables 1-6 are available in the Supplementary Files.

\section{Figures}




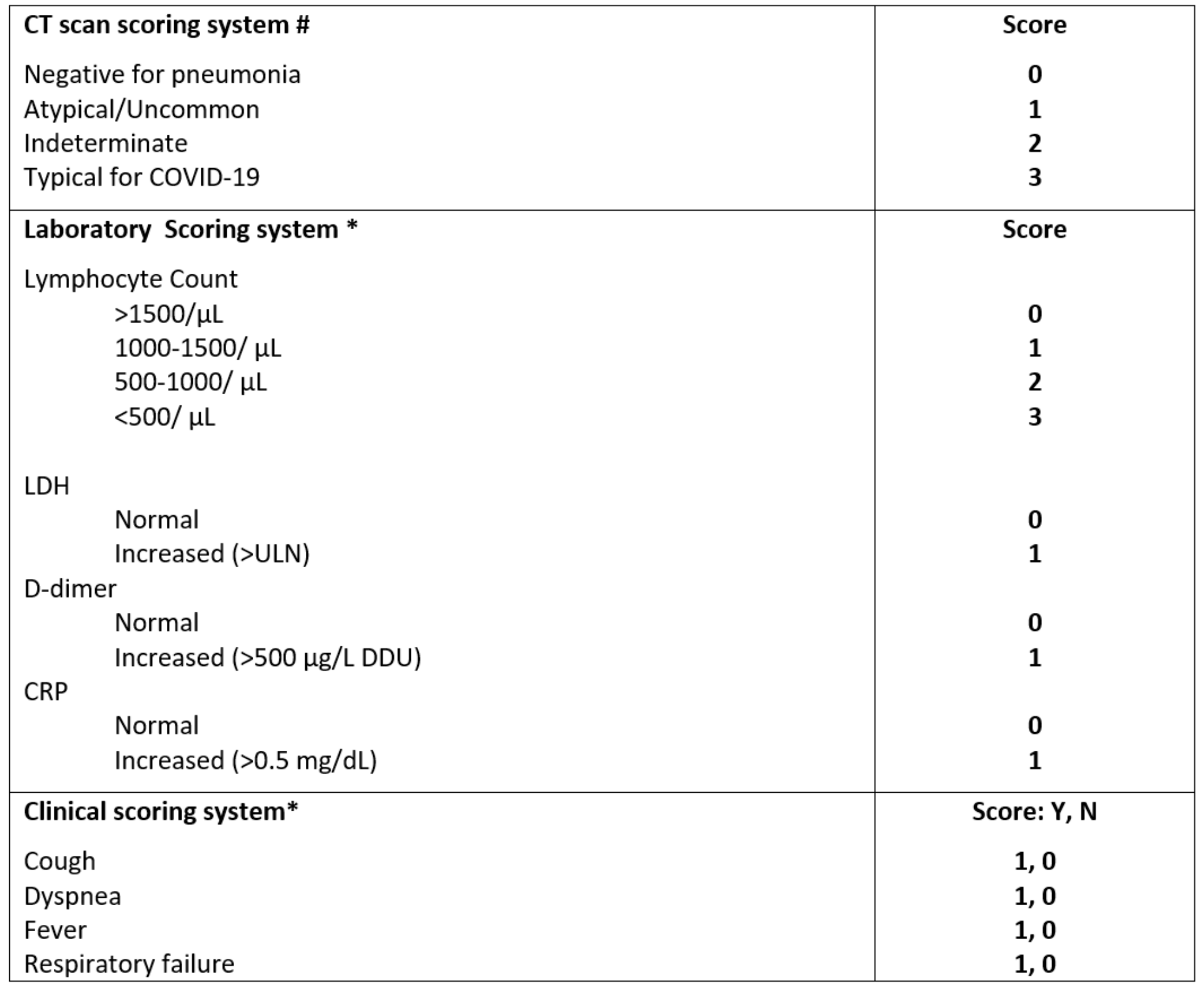

Figure 1

$\mathrm{CT}$ scan, laboratory and clinical scoring system. $\mathrm{LDH}=$ lactate dehydrogenase, $\mathrm{CRP}=\mathrm{C}$ reactive protein, ULN= upper limit of normality, DDU= D-dimer Unit, $\mathrm{Y}=\mathrm{yes}, \mathrm{N}=$ no

\section{Supplementary Files}

This is a list of supplementary files associated with this preprint. Click to download.

- BLCovidTable1290121.docx

- BLCovidTable2310121.docx

- BLCovidTable3290121.docx

- BLCovidTable4290121.docx 
- BLCovidTable5290121.docx

- BLCovidTable6290121.docx 